\title{
Distribution of an Oxytetracycline Resistance Determinant tet(34) among Marine Bacterial Isolates of a Vibrio species
}

\author{
SEOK-RYel Kim ${ }^{1}$, Lisa NonaKa ${ }^{1}$, MyUnG-JoO OH${ }^{2}$, Celia R. LAVILla-PitogO ${ }^{3}$ and SATORU SuZUKi ${ }^{1 *}$ \\ ${ }^{1}$ Center for Marine Environmental Studies (CMES), Ehime University, Matsuyama 790-8577, Japan \\ ${ }^{2}$ Dept. of Aqualife Medicine, Yosu University, Yosu, 550-749, Korea \\ ${ }^{3}$ Southeast Asian Fisheries Development Center (SEAFDEC), Tigbauan, Iloilo, Philippines
}

(Received December 13, 2002-Accepted March 14, 2003)

The distribution of tet(34) was examined among oxytetracycline (OTC) resistant Vibrio strains isolated from Japan, Korea and the Philippines. tet(34) was detected in 10 isolates (6 in fish, 4 in seawater) from Japan and Korea, suggesting that it is widely distributed among fish and seawater bacteria in these countries. Ninety-eight percent of the Vibrio strains in the Philippines were sensitive to OTC, but none of the 60 isolates from the Philippines. The minimum inhibitory concentration (MIC) of OTC-resistant isolates increased 2 to 8 fold in the presence of $\mathrm{Mg}^{2+}$, and MICs were higher in the Japanese and Korean isolates than the Philippine isolates. The MIC for furaltadone (FD) was low in Japanese (23.9\%) isolates at over $3.1 \mu \mathrm{g} / \mathrm{ml}$ and high in Korean $(50 \%)$ and Philippine (56.7\%) isolates. This was probably due to the different frequency of use of FD in these countries. Sequences of $16 \mathrm{~S}$ rDNA of $\operatorname{tet}(34)$-positive isolates were $100 \%$ identical, suggesting that tet(34) is conveyed in a particular Vibrio species.

Key words: Oxytetracycline, tet(34), Vibrio, 16S rRNA, Furaltadone

Oxytetracycline (OTC) is one of the most widely used antibiotics in aquaculture. OTC-resistant bacteria are found in aquaculture sites every year in Japan. A variety of OTCresistant bacteria have been identified in not only humans and animals but also marine environments ${ }^{18,21)}$. OTC resistance determinants are widespread among bacterial species around the world ${ }^{8,10)}$. To date, 36 different tetracycline resistance genes (tet) including OTC resistance genes (otr) have been registered in GenBank. In addition, it is well known that the resistance determinants can be transferred among bacteria by plasmids, transposons and integrons in aquatic environments ${ }^{1,5,19,24)}$. In a previous study, a new OTC resistance determinant tet(34) gave high resistance to the recipient host $E$. coli against both tetracycline and $\mathrm{OTC}^{15)}$. tet(34) is suspected of having a new resistance mechanism, which is associated with xanthine-guanine phosphoribosyl trans-

\footnotetext{
* Corresponding author; E-mail: ssuzuki $@$ agr.ehime-u.ac.jp, Tel: +81-89-927-8552, Fax: +81-89-927-8552

Abbreviations: MIC, minimum inhibitory concentration; TC, tetracycline; OTC, oxytetracycline; FD, furaltadone.
}

ferase (XPRT)-like activity ${ }^{15)}$. $\operatorname{tet}(34)$ has been cloned from Vibrio sp. strain No. 6 isolated from the intestinal content of cultured yellowtail Seriola quinqueradiata in Japan ${ }^{15)}$. tet(34) has been found distributed in certain Vibrio species but not in other fish-related bacteria such as Edwardsiella tarda, Lactococcus garvieae and the Photobacterium damsela subsp. pisicicida ${ }^{16}$.

The first aim of the present study is to clarify the distribution of tet(34) in OTC-resistant bacteria collected from Japan, Korea and the Philippines. Moreover, the genetic relationship is examined among the bacteria possessing tet(34) based on the 16S rDNA sequence.

Furthermore, to examine the resistance of the OTC-resistant bacteria to other drugs, we monitored susceptibility to furaltadon (FD) in all isolates. The incidence of individual and multiple resistance to antibiotic agents has been shown to be associated with the history of antibiotic use ${ }^{2,14)}$. The furans are seldom used in Japan, but in Korea and the Philippines, nitrofuran, furazolidone and FD are used for bacterial chemotherapy $y^{3,6,7,17,23)}$. 


\section{Materials and Methods}

\section{OTC-resistant bacteria isolated in Japan}

Table 1 shows the 46 OTC-resistant Vibrio strains, including 23 isolates obtained from healthy yellowtail Seriola quinqueradiata, 21 isolates from seawater, and 4 isolates from sediment of a farm in Ehime Prefecture, Japan, in 2000. The isolates were identified as Vibrio sp. by the Gram stain method, pigment, and results of the oxidation and fermentation (OF) test and cytochrome oxidase test. They were cultured at $25^{\circ} \mathrm{C}$ in nutrient marine agar plates containing $5 \mathrm{~g}$ of Polypepton (Wako Pure chemical industries, Ltd, Osaka), $1 \mathrm{~g}$ of yeast extract (Difco, Detroit, MI, USA), $1 \mathrm{~g}$ of proteose peptone (Difco), $1 \mathrm{~g}$ of beef extract (Wako Pure chemical industries, Ltd, Osaka), $7.5 \mathrm{~g}$ of Bacto agar (Difco) and $20 \mathrm{~g}$ of $\mathrm{NaCl}$ per liter of distilled water. OTCresistant bacteria classified to the genus Vibrio have a minimum inhibitory concentration (MIC) of $62.5 \mu \mathrm{g} / \mathrm{ml}$ or more.

\section{Bacteria isolated in Korea and the Philippines}

Table 2 shows the 58 Vibrio strains isolated in Korea, including 29 isolates from healthy and diseased fish in farms and 29 isolates from seawater, between 2000 and 2002. Table 3 shows the 60 Vibrio strains of isolated from a hatchery in Iloilo, the Philippines in 2001, including 7 isolates from the eggs of mud crab Scylla sp. collected at a shrimp farm, 32 isolates from the larval stages of shrimp, 14 isolates from nearshore sediment, and 7 isolates from water used to culture the shrimp. Since the MIC for OTC has not been determined for these strains, susceptibility was tested before the tet(34) examination.

\section{Determining the minimum inhibitory concentration (MIC)}

The MICs for all strains were measured as follows. Nutrient marine agar plates ${ }^{15}$ ) were used for the Vibrio isolates. To test whether $\mathrm{Mg}^{2+}$ was needed for the strains to become OTC-resistant, nutrient salt broths with $10 \mathrm{mM}$ of $\mathrm{MgCl}_{2}$ and broths with no $\mathrm{MgCl}_{2}$ were used. A bacterial cell suspension was prepared in phosphate-buffered saline (PBS), and the cell density was adjusted to MacFarland No. 0.5. One microliter portions of the suspensions were then spotted on agar plates containing $31.3,62.5,125,250$ or $500 \mu \mathrm{g} \mathrm{OTC} / \mathrm{ml}$ and $3.1,9.4$ or $18.8 \mu \mathrm{g} / \mathrm{ml}$ of FD. The plates were incubated at $25^{\circ} \mathrm{C}$ for $48 \mathrm{~h}$, and the lowest concentration needed for inhibition was recorded. OTC and FD were obtained from the Sigma Chemical Co, USA.

\section{Genomic DNA extraction}

Genomic DNA was extracted from the OTC-resistant strains and control strains as follows. First, a bacteria cell pellet was suspended in an extraction solution $(0.15 \mathrm{M}$ $\mathrm{NaCl}, 0.1 \mathrm{M}$ EDTA, $0.5 \mathrm{mg} / \mathrm{ml}$ RNase A and 0.5\% SDS) and incubated at $65^{\circ} \mathrm{C}$ for $5 \mathrm{~min}$. The DNA was purified by extraction with phenol saturated with TE buffer [10 mM Tris-HCl (pH 8.0) and $1 \mathrm{mM}$ EDTA]. The DNA was further extracted twice with a TE-saturated phenol:chloroform: iso-amyl alcohol (25:24:1, v/v/v) solution, and once with chloroform. The DNA was finally precipitated using ethanol. Plasmid extraction was performed according to the method of Sambrook and Russell ${ }^{20)}$. Purified chromosome and plasmid DNA was resuspended in $50 \mu \mathrm{l}$ of TE buffer and stored at $-20^{\circ} \mathrm{C}$.

\section{Polymerase chain reaction (PCR) amplification}

To identify the tet(34) in extracted DNA, PCR was performed. The forward primer was 5'-GCTTGCGATTAATTGGTTCC-3' and reverse primer was 5'-TTGGGACGCCATGCAAAATT-3'. This primer set amplified 434 bp from position 314 to 747 of $\operatorname{tet}(34)$. The PCR mixture for tet(34) (total volume $25 \mu \mathrm{l}$ ) contained $20 \mathrm{pmol}$ of each primer, Ex Taq reaction buffer (TaKaRa, Japan), $100 \mu \mathrm{M}$ of dNTPs and 1.0 U of Ex Taq DNA polymerase (TaKaRa, Japan). PCR amplification was performed with a thermal cycler TP-3000 PCR system (TaKaRa, Japan). A single cycle in the amplification program included $95^{\circ} \mathrm{C}$ for $1 \mathrm{~min}$, $55^{\circ} \mathrm{C}$ for $1 \mathrm{~min}$, and $72^{\circ} \mathrm{C}$ for $1 \mathrm{~min}$. A total of 25 cycles were performed, and a final extension was performed at $72^{\circ} \mathrm{C}$ for $5 \mathrm{~min}$. The PCR products were visualized by electrophoresis on a $1.2 \%$ agarose gel (Invitrogen, USA) stained with ethidium bromide.

\section{DNA sequencing and analysis}

To investigate the 16S rDNA of the tet(34)-positive strains, a 1451 bp long PCR product was sequenced. The position was between 8 and 1512 (Eschrichia coli numbering ${ }^{26)}$. The fD1 primer was 5'-AGAGTTTGATCCTGGCTCAG-3' and rP2 primer was 5'-ACGGCTACCTTGTTACGACTT-3'. Forward primers used in this study spanned positions (E. coli numbering) as follows; 5'-CCTACGGGAGGCAGCAG-3' (341 to 357) and 5'-CACAAGCGGTGGAGCATGT-3' (933 to 1011). Reverse primers included 5'-GGCCCGGGAACGTATTCACC-3' (1389 to 1370 ) and 5'-ATTACCGCGGCTGCTGG-3' (534 to 518). Conditions consisted of 30 cycles of $95^{\circ} \mathrm{C}(2 \mathrm{~min}), 42^{\circ} \mathrm{C}(30$ sec) and $72^{\circ} \mathrm{C}(4 \mathrm{~min})$, plus one additional cycle with a final 
Table 1. Minimum inhibitory concentration (MIC) and tet(34) detection in Vibrio sp. isolated from intestinal content of yellowtail and seawater at Uwajima, Japan, in 2000.

\begin{tabular}{|c|c|c|c|c|c|c|c|}
\hline \multirow{2}{*}{ Strain } & \multirow{2}{*}{ Origin } & \multirow{2}{*}{ Area } & \multirow{2}{*}{ Year } & \multicolumn{2}{|c|}{ MIC ( $\mu \mathrm{g}$ OTC/ml) } & \multirow{2}{*}{$\mathrm{MIC}(\mu \mathrm{g} \mathrm{FD} / \mathrm{ml})$} & \multirow{2}{*}{$\operatorname{tet}(34)$} \\
\hline & & & & without $\mathrm{Mg}^{2+}$ & with $\mathrm{Mg}^{2+}$ & & \\
\hline $1-\mathrm{A}$ & Yellowtail (I) & Uwajima & 2000.10 & 62.5 & 125 & 3.1 & - \\
\hline $1-\mathrm{C}$ & Yellowtail (I) & Uwajima & 2000.10 & 62.5 & 250 & $<3.1$ & - \\
\hline $1-\mathrm{D}$ & Yellowtail (I) & Uwajima & 2000.10 & 62.5 & 250 & $<3.1$ & - \\
\hline $1-\mathrm{F}$ & Yellowtail (I) & Uwajima & 2000.10 & 62.5 & $>500$ & 3.1 & - \\
\hline $1-G$ & Yellowtail (I) & Uwajima & 2000.10 & 62.5 & $>500$ & 3.1 & + \\
\hline $1-\mathrm{H}$ & Yellowtail (I) & Uwajima & 2000.10 & 62.5 & $>500$ & 3.1 & - \\
\hline $2-\mathrm{A}$ & Yellowtail (I) & Uwajima & 2000.10 & 62.5 & 250 & $<3.1$ & - \\
\hline $2-\mathrm{H}$ & Yellowtail (I) & Uwajima & 2000.10 & 62.5 & 125 & $<3.1$ & + \\
\hline $2-\mathrm{I}$ & Yellowtail (I) & Uwajima & 2000.10 & 62.5 & 250 & $<3.1$ & + \\
\hline 2-J & Yellowtail (I) & Uwajima & 2000.10 & 62.5 & 250 & $<3.1$ & - \\
\hline $3-\mathrm{A}$ & Yellowtail (I) & Uwajima & 2000.10 & 62.5 & 250 & 3.1 & - \\
\hline $3-\mathrm{C}$ & Yellowtail (I) & Uwajima & 2000.10 & 62.5 & 250 & 3.1 & - \\
\hline $3-\mathrm{E}$ & Yellowtail (I) & Uwajima & 2000.10 & 62.5 & $>500$ & $<3.1$ & - \\
\hline $3-\mathrm{G}$ & Yellowtail (I) & Uwajima & 2000.10 & 250 & 250 & 3.1 & - \\
\hline 3-J & Yellowtail (I) & Uwajima & 2000.10 & 250 & $>500$ & $<3.1$ & - \\
\hline $4-\mathrm{A}$ & Yellowtail (I) & Uwajima & 2000.10 & 250 & $>500$ & $<3.1$ & - \\
\hline $4-\mathrm{B}$ & Yellowtail (I) & Uwajima & 2000.10 & 250 & $>500$ & $<3.1$ & - \\
\hline $4-\mathrm{E}$ & Yellowtail (I) & Uwajima & 2000.10 & 250 & $>500$ & $<3.1$ & - \\
\hline $4-\mathrm{F}$ & Yellowtail (I) & Uwajima & 2000.10 & 250 & $>500$ & $<3.1$ & - \\
\hline $4-G$ & Yellowtail (I) & Uwajima & 2000.10 & 62.5 & 125 & $<3.1$ & - \\
\hline $4-\mathrm{H}$ & Yellowtail (I) & Uwajima & 2000.10 & 250 & $>500$ & $<3.1$ & - \\
\hline $5-A$ & Yellowtail (I) & Uwajima & 2000.10 & 62.5 & 125 & $<3.1$ & + \\
\hline $5-\mathrm{B}$ & Yellowtail (I) & Uwajima & 2000.10 & 62.5 & 250 & $<3.1$ & - \\
\hline Sw-1 & seawater & Uwajima & 2000.10 & 125 & 250 & $<3.1$ & - \\
\hline Sw-5 & seawater & Uwajima & 2000.10 & 62.5 & 250 & $<3.1$ & - \\
\hline Sw-6 & seawater & Uwajima & 2000.10 & 62.5 & 250 & $<3.1$ & - \\
\hline Sw-7 & seawater & Uwajima & 2000.10 & 62.5 & 250 & $<3.1$ & - \\
\hline Sw-8 & seawater & Uwajima & 2000.10 & 62.5 & 125 & $<3.1$ & - \\
\hline Sw-11 & seawater & Uwajima & 2000.10 & 250 & $>500$ & $<3.1$ & - \\
\hline Sw-14 & seawater & Uwajima & 2000.10 & 62.5 & $>500$ & $<3.1$ & - \\
\hline Sw-16 & seawater & Uwajima & 2000.10 & 62.5 & $>500$ & $<3.1$ & - \\
\hline Sw-18 & seawater & Uwajima & 2000.10 & 250 & $>500$ & $<3.1$ & - \\
\hline Sw-32 & seawater & Uwajima & 2000.10 & 250 & $>500$ & $<3.1$ & - \\
\hline Sw-34 & seawater & Uwajima & 2000.10 & 62.5 & 250 & $<3.1$ & - \\
\hline Sw-37 & seawater & Uwajima & 2000.10 & 125 & $>500$ & $<3.1$ & - \\
\hline Sw-38 & seawater & Uwajima & 2000.10 & 125 & $>500$ & $<3.1$ & - \\
\hline Sw-41 & seawater & Uwajima & 2000.10 & 125 & $>500$ & $<3.1$ & - \\
\hline Sw-42 & seawater & Uwajima & 2000.10 & 125 & $>500$ & $<3.1$ & + \\
\hline Sw-44 & seawater & Uwajima & 2000.10 & 62.5 & $>500$ & 18.8 & - \\
\hline Sw-48 & seawater & Uwajima & 2000.10 & 125 & $>500$ & $<3.1$ & - \\
\hline Sw-50 & seawater & Uwajima & 2000.10 & 125 & $>500$ & 3.1 & - \\
\hline Sw-a & seawater & Uwajima & 2000.10 & 250 & $>500$ & 3.1 & - \\
\hline $\mathrm{Sd}-2$ & sediment & Uwajima & 2000.10 & 62.5 & 250 & $<3.1$ & - \\
\hline $\mathrm{Sd}-7$ & sediment & Uwajima & 2000.10 & 62.5 & $>500$ & 3.1 & - \\
\hline $\mathrm{Sd}-8$ & sediment & Uwajima & 2000.10 & 62.5 & 125 & 3.1 & - \\
\hline Sd-18 & sediment & Uwajima & 2000.10 & 125 & $>500$ & $<3.1$ & - \\
\hline No. 6 & \multicolumn{3}{|c|}{ Positive control \{origin strain of tet(34)\}; Vibrio sp. } & 125 & $>500$ & $<3.1$ & + \\
\hline ATCC $17802 \mathrm{~T}$ & \multicolumn{3}{|c|}{$\begin{array}{l}\text { Non-fish strain negative control (sentitive to OTC); } \\
\text { Vibrio parahaemolyticus }\end{array}$} & $<31.3$ & $<31.3$ & $<3.1$ & - \\
\hline
\end{tabular}

(I); intestinal content 
Table 2. Minimum inhibitory concentration (MIC) and tet(34) detection in Vibrio sp. isolated from diseased fish and seawater in south Korea.

\begin{tabular}{|c|c|c|c|c|c|c|c|}
\hline \multirow{2}{*}{ Strain } & \multirow{2}{*}{ Origin } & \multirow{2}{*}{ Area } & \multirow{2}{*}{ Year } & \multicolumn{2}{|c|}{$\mathrm{MIC}(\mu \mathrm{g} \mathrm{OTC} / \mathrm{ml})$} & \multirow{2}{*}{$\mathrm{MIC}(\mu \mathrm{g} \mathrm{FD} / \mathrm{ml})$} & \multirow{2}{*}{$\operatorname{tet}(34)$} \\
\hline & & & & without $\mathrm{Mg}^{2+}$ & with $\mathrm{Mg}^{2+}$ & & \\
\hline FK0001 & Flounder $(\mathrm{K})$ & Yosu & 2000.1 & $<31.3$ & $<31.3$ & 3.1 & - \\
\hline SK0102 & Sea bass (K) & Yosu & 2001.2 & $<31.3$ & $<31.3$ & $<3.1$ & - \\
\hline RFK01041 & Rock fish $(\mathrm{K})$ & Yosu & 2001.4 & $<31.3$ & $<31.3$ & $<3.1$ & - \\
\hline RFS01042 & Rock fish (S) & Yosu & 2001.4 & $<31.3$ & $<31.3$ & $<3.1$ & - \\
\hline FK01073 & Flounder (K) & Wando & 2001.7 & 125 & 250 & $<3.1$ & + \\
\hline FK0103 & Flounder $(\mathrm{K})$ & Youngkwang & 2001.3 & $<31.3$ & $<31.3$ & $<3.1$ & - \\
\hline FK01071 & Flounder (K) & Youngkwang & 2001.7 & $<31.3$ & $<31.3$ & $<3.1$ & - \\
\hline FK01072 & Flounder (K) & Youngkwang & 2001.7 & 62.5 & 250 & 9.4 & - \\
\hline FI0203 & Flounder (I) & Youngkwang & 2002.2 & $<31.3$ & $<31.3$ & $<3.1$ & - \\
\hline FI0204 & Flounder (I) & Youngkwang & 2002.2 & 62.5 & 250 & 3.1 & + \\
\hline FI0205 & Flounder (I) & Youngkwang & 2002.2 & 125 & 500 & $<3.1$ & - \\
\hline FI0206 & Flounder (I) & Youngkwang & 2002.2 & 62.5 & 250 & 3.1 & - \\
\hline FI0207 & Flounder (I) & Youngkwang & 2002.2 & $<31.3$ & $<31.3$ & 18.8 & - \\
\hline FI0208 & Flounder (I) & Youngkwang & 2002. 2 & $<31.3$ & 31.3 & $<3.1$ & - \\
\hline FI0209 & Flounder (I) & Youngkwang & 2002.2 & 125 & $>500$ & 3.1 & - \\
\hline RI0210 & Rock bream (I) & Youngkwang & 2002.2 & $<31.3$ & 125 & 3.1 & - \\
\hline RI0211 & Rock bream (I) & Youngkwang & 2002.2 & 31.3 & 125 & 3.1 & - \\
\hline RI0212 & Rock bream (I) & Youngkwang & 2002.2 & $<31.3$ & $<31.3$ & 3.1 & - \\
\hline FI0213 & Flounder (K) & Wando & 2002.2 & $<31.3$ & $<31.3$ & $<3.1$ & - \\
\hline FI0214 & Flounder (K) & Jindo & 2002.2 & $<31.3$ & $<31.3$ & $<3.1$ & - \\
\hline FI0215 & Flounder (K) & Jindo & 2002. 2 & $<31.3$ & $<31.3$ & $<3.1$ & - \\
\hline FI0216 & Flounder (I) & Jindo & 2002.2 & $<31.3$ & $<31.3$ & $<3.1$ & - \\
\hline FI0217 & Flounder (I) & Jindo & 2002.2 & $<31.3$ & $<31.3$ & 3.1 & - \\
\hline FI0218 & Flounder (I) & Jindo & 2002.2 & $<31.3$ & $<31.3$ & 3.1 & - \\
\hline FI0219 & Flounder (I) & Jindo & 2002.2 & $<31.3$ & $<31.3$ & 31.3 & - \\
\hline FI0220 & Flounder (I) & Jindo & 2002.2 & $<31.3$ & $<31.3$ & $<3.1$ & - \\
\hline FI0221 & Flounder (I) & Jindo & 2002.2 & $<31.3$ & $<31.3$ & 18.8 & - \\
\hline FI0222 & Flounder (I) & Jindo & 2002.2 & $<31.3$ & 62.5 & 3.1 & - \\
\hline FI0223 & Flounder (I) & Jindo & 2002.2 & $<31.3$ & $<31.3$ & $<3.1$ & - \\
\hline SMI0224 & Striped mullet (I) & Mokpo & 2002.2 & $<31.3$ & $<31.3$ & $<3.1$ & - \\
\hline SMI0225 & Striped mullet (I) & Mokpo & 2002. 2 & $<31.3$ & $<31.3$ & 3.1 & - \\
\hline SW01071 & Sea water & Youngkwang & 2001.7 & $<31.3$ & 31.3 & 3.1 & - \\
\hline SW01072 & Sea water & Youngkwang & 2001.7 & 125 & $>500$ & 3.1 & - \\
\hline SW01073 & Sea water & Youngkwang & 2001.7 & 250 & $>500$ & 31.3 & - \\
\hline SW01074 & Sea water & Youngkwang & 2001.7 & $<31.3$ & 31.3 & 3.1 & - \\
\hline SW01075 & Sea water & Youngkwang & 2001.7 & $<31.3$ & $<31.3$ & 3.1 & - \\
\hline SW01076 & Sea water & Youngkwang & 2001.7 & 125 & $>500$ & 3.1 & - \\
\hline SW0201 & Sea water & Youngkwang & 2002.2 & 31.3 & 250 & 3.1 & - \\
\hline SW0202 & Sea water & Youngkwang & 2002.2 & 125 & 250 & 3.1 & + \\
\hline SW0203 & Sea water & Youngkwang & 2002.2 & 62.5 & 250 & 3.1 & - \\
\hline SW0204 & Sea water & Youngkwang & 2002.2 & $<31.3$ & $<31.3$ & $<3.1$ & - \\
\hline SW0205 & Sea water & Youngkwang & 2002.2 & $<31.3$ & $<31.3$ & $<3.1$ & - \\
\hline SW0206 & Sea water & Youngkwang & 2002.2 & 125 & 250 & 3.1 & + \\
\hline SW0207 & Sea water & Youngkwang & 2002. 2 & $<31.3$ & $<31.3$ & $<3.1$ & - \\
\hline SW0208 & Sea water & Youngkwang & 2002.2 & $<31.3$ & $<31.3$ & $<3.1$ & - \\
\hline SW0209 & Sea water & Youngkwang & 2002.2 & $<31.3$ & 31.3 & $<3.1$ & - \\
\hline SW0210 & Sea water & Youngkwang & 2002.2 & 125 & 250 & $<3.1$ & - \\
\hline SW0211 & Sea water & Youngkwang & 2002.2 & $<31.3$ & 31.3 & 3.1 & - \\
\hline SW0212 & Sea water & Youngkwang & 2002.2 & 125 & 250 & $<3.1$ & + \\
\hline SW0213 & Sea water & Youngkwang & 2002.2 & $<31.3$ & 62.5 & $<3.1$ & - \\
\hline SW0214 & Sea water & Mokpo & 2002.2 & $<31.3$ & $<31.3$ & $<3.1$ & - \\
\hline SW0215 & Sea water & Mokpo & 2002.2 & $<31.3$ & $<31.3$ & $<3.1$ & - \\
\hline SW0216 & Sea water & Jindo & 2002.2 & 62.5 & 250 & $<3.1$ & - \\
\hline SW0217 & Sea water & Jindo & 2002.2 & $<31.3$ & $<31.3$ & 3.1 & - \\
\hline SW0218 & Sea water & Jindo & 2002.2 & $<31.3$ & $<31.3$ & $<3.1$ & - \\
\hline SW0219 & Sea water & Yosu & 2002.2 & $<31.3$ & $<31.3$ & $<3.1$ & - \\
\hline SW0220 & Sea water & Yosu & 2002.2 & $<31.3$ & $<31.3$ & 3.1 & - \\
\hline SW0221 & Sea water & Yosu & 2002.2 & $<31.3$ & $<31.3$ & 3.1 & - \\
\hline
\end{tabular}

(K) kidney; (S), spleen and (I), intestinal content 
Table 3. Minimum inhibitory concentration (MIC) and tet(34) detection in Vibrio sp. isolated from crustaceans and the environment at Iloilo, the Philippines, in 2001.

\begin{tabular}{|c|c|c|c|c|c|c|c|}
\hline \multirow{2}{*}{ Strain } & \multirow{2}{*}{ Origin } & \multirow{2}{*}{ Area } & \multirow{2}{*}{ Year } & \multicolumn{2}{|c|}{$\mathrm{MIC}(\mu \mathrm{g} \mathrm{OTC} / \mathrm{ml})$} & \multirow{2}{*}{$\mathrm{MIC}(\mu \mathrm{g} \mathrm{FD} / \mathrm{ml})$} & \multirow{2}{*}{$\operatorname{tet}(34)$} \\
\hline & & & & without $\mathrm{Mg}^{2+}$ & with $\mathrm{Mg}^{2+}$ & & \\
\hline $\mathrm{O} 1 \mathrm{~S} 2{ }_{3}$ & Egg: Scylla serrata & Iloilo & 2001.5 & $<31.3$ & $<31.3$ & 9.4 & - \\
\hline $\mathrm{O}_{1 T} 1_{1}$ & Egg: Scylla tranquebarica & Iloilo & 2001.5 & $<31.3$ & $<31.3$ & $<3.1$ & - \\
\hline $\mathrm{O}_{1 T} 1_{2}$ & Egg: Scylla tranquebarica & Iloilo & 2001.5 & $<31.3$ & $<31.3$ & $<3.1$ & - \\
\hline $\mathrm{O}_{1 T} 1_{3}$ & Egg: Scylla tranquebarica & Iloilo & 2001.5 & $<31.3$ & $<31.3$ & $<3.1$ & - \\
\hline $\mathrm{S} 4_{1}$ & Egg: Scylla serrata & Iloilo & 2001.5 & $<31.3$ & $<31.3$ & 3.1 & - \\
\hline $\mathrm{O}_{5}$ & Egg: Scylla olivacea & Iloilo & 2001.5 & $<31.3$ & $<31.3$ & 3.1 & - \\
\hline $\mathrm{U}$ & Egg: Unablated Scylla serrata & Iloilo & 2001.5 & $<31.3$ & $<31.3$ & $<3.1$ & - \\
\hline $\mathrm{Z} 1-1$ & Zoea in crustacean hatchery & Iloilo & 2001.5 & $<31.3$ & $<31.3$ & $<3.1$ & - \\
\hline Z1-2 & Zoea in crustacean hatchery & Iloilo & 2001.5 & $<31.3$ & $<31.3$ & $<3.1$ & - \\
\hline $\mathrm{Z2}-1$ & Zoea in crustacean hatchery & Iloilo & 2001.5 & 31.3 & 125 & 3.1 & - \\
\hline $\mathrm{Z} 2-2$ & Zoea in crustacean hatchery & Iloilo & 2001.5 & $<31.3$ & $<31.3$ & $<3.1$ & - \\
\hline Z3-1 & Zoea in crustacean hatchery & Iloilo & 2001.5 & $<31.3$ & $<31.3$ & 3.1 & - \\
\hline Z3-2 & Zoea in crustacean hatchery & Iloilo & 2001.5 & $<31.3$ & $<31.3$ & 9.4 & - \\
\hline Z4-1 & Zoea in crustacean hatchery & Iloilo & 2001.5 & $<31.3$ & $<31.3$ & $<3.1$ & - \\
\hline $\mathrm{Z} 4-2$ & Zoea in crustacean hatchery & Iloilo & 2001.5 & $<31.3$ & $<31.3$ & $<3.1$ & - \\
\hline ZT3-1 & Zoea in crustacean hatchery & Iloilo & 2001.5 & $<31.3$ & $<31.3$ & $<3.1$ & - \\
\hline ZT3-2 & Zoea in crustacean hatchery & Iloilo & 2001.5 & $<31.3$ & $<31.3$ & $<3.1$ & - \\
\hline ZT4-1 & Zoea in crustacean hatchery & Iloilo & 2001.5 & $<31.3$ & $<31.3$ & $<3.1$ & - \\
\hline ZT4-2 & Zoea in crustacean hatchery & Iloilo & 2001.5 & $<31.3$ & $<31.3$ & $<3.1$ & - \\
\hline ZT6-1 & Zoea in crustacean hatchery & Iloilo & 2001.5 & $<31.3$ & $<31.3$ & $<3.1$ & - \\
\hline ZT6-2 & Zoea in crustacean hatchery & Iloilo & 2001.5 & $<31.3$ & $<31.3$ & $<3.1$ & - \\
\hline ZT11-1 & Zoea in crustacean hatchery & Iloilo & 2001.5 & $<31.3$ & $<31.3$ & $<3.1$ & - \\
\hline ZT11-2 & Zoea in crustacean hatchery & Iloilo & 2001.5 & $<31.3$ & $<31.3$ & $<3.1$ & - \\
\hline Z1-1 & Zoea in crustacean hatchery & Iloilo & 2001.5 & $<31.3$ & $<31.3$ & $<3.1$ & - \\
\hline $\mathrm{Z1-2}$ & Zoea in crustacean hatchery & Iloilo & 2001.5 & $<31.3$ & $<31.3$ & $<3.1$ & - \\
\hline $\mathrm{Z} 2-1$ & Zoea in crustacean hatchery & Iloilo & 2001.5 & $<31.3$ & $<31.3$ & $<3.1$ & - \\
\hline $\mathrm{Z} 2-2$ & Zoea in crustacean hatchery & Iloilo & 2001.5 & $<31.3$ & $<31.3$ & $<3.1$ & - \\
\hline Z3-1 & Zoea in crustacean hatchery & Iloilo & 2001.5 & $<31.3$ & $<31.3$ & $<3.1$ & - \\
\hline Z3-2 & Zoea in crustacean hatchery & Iloilo & 2001.5 & $<31.3$ & $<31.3$ & 3.1 & - \\
\hline Z4-1 & Zoea in crustacean hatchery & Iloilo & 2001.5 & $<31.3$ & $<31.3$ & 3.1 & - \\
\hline Z4-2 & Zoea in crustacean hatchery & Iloilo & 2001.5 & $<31.3$ & $<31.3$ & $<3.1$ & - \\
\hline ZT3-1 & Zoea in crustacean hatchery & Iloilo & 2001.5 & $<31.3$ & $<31.3$ & $<3.1$ & - \\
\hline ZT3-2 & Zoea in crustacean hatchery & Iloilo & 2001.5 & $<31.3$ & $<31.3$ & 3.1 & - \\
\hline ZT4-1 & Zoea in crustacean hatchery & Iloilo & 2001.5 & $<31.3$ & $<31.3$ & 9.4 & - \\
\hline ZT4-2 & Zoea in crustacean hatchery & Iloilo & 2001.5 & $<31.3$ & $<31.3$ & 3.1 & - \\
\hline ZT6-1 & Zoea in crustacean hatchery & Iloilo & 2001.5 & $<31.3$ & $<31.3$ & 3.1 & - \\
\hline ZT6-2 & Zoea in crustacean hatchery & Iloilo & 2001.5 & $<31.3$ & $<31.3$ & 3.1 & - \\
\hline ZT11-1 & Zoea in crustacean hatchery & Iloilo & 2001.5 & $<31.3$ & $<31.3$ & $<3.1$ & - \\
\hline ZT11-2 & Zoea in crustacean hatchery & Iloilo & 2001.5 & $<31.3$ & $<31.3$ & 3.1 & - \\
\hline Sdt1 & Nearshore sediment & Iloilo & 2001.5 & $<31.3$ & $<31.3$ & 3.1 & - \\
\hline $\mathrm{SW} 1_{1}$ & Nearshore sediment & Iloilo & 2001.5 & $<31.3$ & $<31.3$ & 3.1 & - \\
\hline $\mathrm{SW}_{2}$ & Nearshore sediment & Iloilo & 2001.5 & $<31.3$ & $<31.3$ & 3.1 & - \\
\hline $\mathrm{SW}_{3}$ & Nearshore sediment & Iloilo & 2001.5 & $<31.3$ & $<31.3$ & 3.1 & - \\
\hline $\mathrm{Sdt} 2{ }_{1}$ & Nearshore sediment & Iloilo & 2001.5 & $<31.3$ & $<31.3$ & 3.1 & - \\
\hline $\mathrm{Sdt} 2_{2}$ & Nearshore sediment & Iloilo & 2001.5 & $<31.3$ & $<31.3$ & 3.1 & - \\
\hline $\mathrm{SW} 2_{1}$ & Nearshore sediment & Iloilo & 2001.5 & $<31.3$ & $<31.3$ & 3.1 & - \\
\hline $\mathrm{SW}_{2}$ & Nearshore sediment & Iloilo & 2001.5 & $<31.3$ & $<31.3$ & 3.1 & - \\
\hline Sdt 31 & Nearshore sediment & Iloilo & 2001.5 & $<31.3$ & $<31.3$ & 3.1 & - \\
\hline $\mathrm{Sdt}_{2}$ & Nearshore sediment & Iloilo & 2001.5 & $<31.3$ & $<31.3$ & 3.1 & - \\
\hline $\mathrm{Sdt}_{3}$ & Nearshore sediment & Iloilo & 2001.5 & $<31.3$ & $<31.3$ & $<3.1$ & - \\
\hline $\mathrm{Sdt}_{4}$ & Nearshore sediment & Iloilo & 2001.5 & $<31.3$ & $<31.3$ & 3.1 & - \\
\hline $\mathrm{Sdt}_{5}$ & Nearshore sediment & Iloilo & 2001.5 & $<31.3$ & $<31.3$ & 3.1 & - \\
\hline Sdt36 & Nearshore sediment & Iloilo & 2001.5 & $<31.3$ & $<31.3$ & 3.1 & - \\
\hline WL1 & Water from zoea & Iloilo & 2001.5 & $<31.3$ & $<31.3$ & 3.1 & - \\
\hline WL2 & Water from zoea & Iloilo & 2001.5 & $<31.3$ & $<31.3$ & 3.1 & - \\
\hline WP2 & Water from 48-day postlarva & Iloilo & 2001.5 & $<31.3$ & $<31.3$ & 3.1 & - \\
\hline WP8 & Water from 48-day postlarva & Iloilo & 2001.5 & $<31.3$ & $<31.3$ & 3.1 & - \\
\hline WP9 & Water from 48-day postlarva & Iloilo & 2001.5 & $<31.3$ & $<31.3$ & 3.1 & - \\
\hline WP11 & Water from 48-day postlarva & Iloilo & 2001.5 & $<31.3$ & $<31.3$ & 3.1 & - \\
\hline WP14 & Water from 48-day postlarva & Iloilo & 2001.5 & $<31.3$ & $<31.3$ & 3.1 & - \\
\hline
\end{tabular}


20 min chain elongation. All of the tet(34)-positive strains (1-G, 2-H, 2-I, 5-A, Sw-42, FK01073, FI0204, SW0202, SW0206 and SW0212) were analyzed. Sequencing was performed using the DNA sequencing Kit (Applied Biosystems, Tokyo) and the ABI PRISM 310 DNA sequencer (Perkin Elmer, Tokyo) at the Center for Gene Research, Ehime University. Searches for similar nucleotide sequences of $16 \mathrm{~S}$ rDNA of tet(34)-positive strains and other strains (Vibrio sp. strain No. 6; GenBank accession No. AB089204, V. scopthalmi; GenBank accession No. U46579, $V$. ichthyoenteri; GenBank accession No. AJ437192 and $V$. vulnificus; GenBank accession No. X76334) were performed using GENETYX-WIN (Ver. 5.0) software.

\section{Results}

\section{MIC for OTC and FD}

The MICs of 46 OTC-resistant Vibrio strains collected from a fish farm in Japan are shown in Table 1. The MIC for OTC was 62.5 to $250 \mu \mathrm{g} / \mathrm{ml}$ when $\mathrm{Mg}^{2+}$ was absent, and 2 times higher when $\mathrm{Mg}^{2+}$ was present. The MIC for FD was generally $3.1 \mu \mathrm{g} / \mathrm{ml}(21.7 \%)$ or less $(76.1 \%)$. One strain had an MIC of $18.8 \mu \mathrm{g} / \mathrm{ml}$.

The MICs of 58 Vibrio strains isolated from marine fish farms in Korea and 60 Vibrio strains isolated from a shrimp hatchery in the Philippines are shown in Table 2 and Table 3 , respectively. In the Korean strains, 15 strains had MICs of $61.5 \mu \mathrm{g} / \mathrm{ml}$ or higher for OTC, and MICs of $31.3 \mu \mathrm{g} / \mathrm{ml}$ or less when $\mathrm{Mg}^{2+}$ was absent. The MIC of most of the sensitive strains with an MIC of $<31.3 \mu \mathrm{g} / \mathrm{ml}$ did not increase even when $\mathrm{Mg}^{2+}$ was added. Most MICs for FD were 3.1 $\mu \mathrm{g} / \mathrm{ml}(41.3 \%)$ or less $(50 \%)$. Five strains had MICs of 9.4 to $31.3 \mu \mathrm{g} / \mathrm{ml}$.

Most of the Philippine strains (98.3\%) were sensitive to OTC. Only one strain had an MIC of $31.3 \mu \mathrm{g} / \mathrm{ml}$. Addition of $\mathrm{Mg}^{2+}$ increased the MIC of strain Z2-1 to $125 \mu \mathrm{g} / \mathrm{ml}$, but caused no increase in the other strains (Table 3). Most MICs for FD were $3.1 \mu \mathrm{g} / \mathrm{ml}(51.6 \%)$ or less $(43.3 \%)$. Three strains had an MIC of $9.4 \mu \mathrm{g} / \mathrm{ml}$. The distribution of the MICs for FD is summarized in Fig. 1.

\section{Detection of tet(34)}

Five of the Japanese strains tested positive for tet(34) (Table 1). Four strains (1-G, 2-H, 2-I and 5-A) were isolated from the intestinal contents of yellowtail, and one strain (Sw-42) was isolated from seawater. The MICs of all tet(34)-positive strains were $>125 \mu \mathrm{g} / \mathrm{ml}$ in the presence of $\mathrm{Mg}^{2+}$ (Table 1).

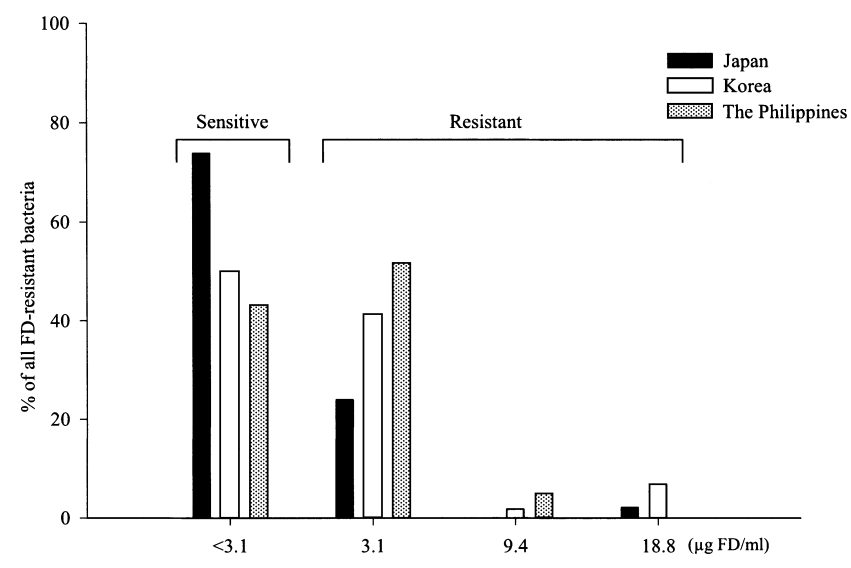

Fig. 1. Occupation rate of minimum inhibitory concentration (MIC) for furaltadone (FD) in Japanese, Korean and Philippine strains.

In the Korean isolates, $6 \operatorname{tet}(34)$-positive strains were identified: strain FK01073 from the kidney of diseased flounder, strain FI0204 from the intestinal contents of healthy flounder, and 3 strains (SW0202, SW0206 and SW0212) from seawater (Table 2). These 6 strains had MICs of 62.5 to $250 \mu \mathrm{g} / \mathrm{ml}$ in the presence of $\mathrm{Mg}^{2+}$. None of the Philippine strains tested positive for tet(34) (Table 3).

\section{Sequences of 16S rDNA of tet(34)-positive strains}

The 16S rDNA of $10 \operatorname{tet}(34)$-positive strains were sequenced. All were $100 \%$ identical to Vibrio sp. strain No. 6, which is an origin strain of $\operatorname{tet}(34)$ (Fig. 2).

\section{Discussion}

The tetracycline (TC) series are effective against a wide range of Gram-positive and Gram-negative bacteria ${ }^{8,11}$, so they have been used extensively as antimicrobial agents in aquaculture. However, bacteria resistant to these drugs are now occurring more frequently ${ }^{1,21,23)}$.

The resistance mechanisms of the bacteria are known based on the chemical properties of the drug. The TC has a number of potential metal-binding sites, and they form a complex with magnesium and calcium ${ }^{4,9,12,13)}$. Lunestad and Goksoyr ${ }^{12)}$ reported that only about $5 \%$ of the OTC exists in its free form. The MIC value of OTC was approximately 10 times higher when $\mathrm{Mg}^{2+}$ or $\mathrm{Ca}^{2+}$ was in the media, indicating that OTC that has formed a complex with either of these ions has a reduced ability to cross lipid-rich biological membranes. Seawater contains many divalent cations, which cause the activity of OTC to decrease in seawater. MIC values for OTC were 2 to 8 times higher than when there was no $\mathrm{Mg}^{2+}$ (Table 1, 2 and 3). These results confirm 


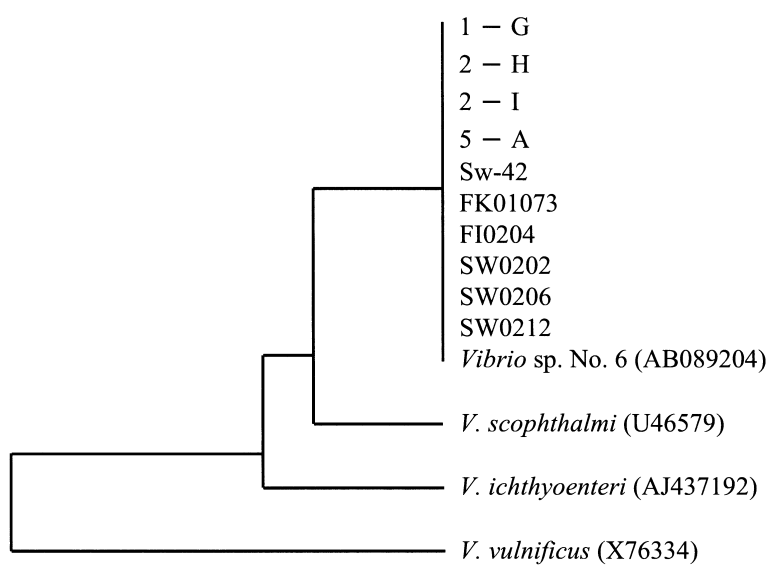

0.01

Fig. 2. Phylogenetic tree based on $16 \mathrm{~S}$ rDNA of tet(34)-positive isolates (unweighted pair group method with arithmetic mean, UPGMA). The number in parentheses indicates the GenBank accession number.

that most OTC resistance in marine bacteria could be enhanced by an $\mathrm{Mg}^{2+}$ associated system ${ }^{15,22)}$. Nonaka and Suzuki ${ }^{15)}$ proposed new resistance mechanism involved in the association of $\mathrm{Mg}^{2+}$ with the $\operatorname{tet}(34)$ product.

Tendencia and de la Peňa ${ }^{23)}$ reported that $4.7 \%$ of bacteria from a shrimp farm in the Philippines were OTC-resistant and the MICs for OTC were $30 \mu \mathrm{g} /$ disc. They reported that the occurrence of OTC resistance was much higher than the occurrence of FD, oxolinic acid and chloramphenicol resistance. In our study, the OTC resistance rate was much lower (1.6\%) than the occurrence rates of FD (57\%) and erythromycin ( $93.3 \%$, data not shown). The mechanisms of bacterial resistance to erythromycin include the modification of ribosomal targets by a methylase associated with transmissible genes of the erm family, various antibiotic efflux mechanisms, and point mutations in the peptidyltransferase region of $23 \mathrm{~S}$ rRNA genes ${ }^{25)}$. This difference might be caused by culture conditions or the drug treatment history of the shrimp.

A total of $26 \%$ of the OTC-resistant bacteria from Japan were resistant to FD, whereas $46 \%$ of the Korean strains and $57 \%$ of the Philippine strains were resistant to FD (Fig. 1). This suggests that resistance to antibiotics is affected by the history of antibiotic use ${ }^{2,14)}$. In Korea and the Philippines, the wide used of FD for various bacterial diseases may have caused this increased resistance. FD is not used in Japan, where FD resistance was lower.

tet(34)-positive strains were detected from the Vibrio strains isolated from both healthy and diseased fish, and from seawater. This suggests that tet(34) was widely spread among fish and seawater.

$\operatorname{tet}(\mathrm{A}), \operatorname{tet}(\mathrm{B}), \operatorname{tet}(\mathrm{C}), \operatorname{tet}(\mathrm{D}), \operatorname{tet}(\mathrm{E})$ and $\operatorname{tet}(\mathrm{G})$ occur in the Vibrio species and other Gram-negative bacteria, suggesting that these genes can be transferred to different bacterial species. This transfer is mediated by many natural mobile elements, including transposons and conjugative plasmids in aquatic ecosystems ${ }^{1,5,8,19}$. The $16 \mathrm{~S}$ rDNA in all tet(34)positive strains was $100 \%$ identical to that of Vibrio sp. strain No. 6, suggesting that tet(34) is present in specific species of Vibrio. Our results clearly show that this bacterium is widely distributed in fish intestines and seawater in Japan and Korea. It was found that tet(34) is conveyed on a chromosome of Vibrio sp. No. $6^{16)}$, suggesting that tet(34) does not transfer to other bacterial species. $\operatorname{tet}(34)$ might differ from the other known tet series in terms of gene transfer. The deduced product of tet(34) is XPRT, which is found in many species such as E. coli. However, E. coli was not resistant to OTC, which suggests that the expression of tet(34) occurs more frequently in the tet(34)-positive Vibrio species than in other bacteria. The molecular properties of tet(34) and the distribution of tet(34)-positive Vibrio sp. will be studied further in future. Since Vibrio sp. strain No. 6 is genetically very similar to $V$. scophthami and $V$. ichthyoenteri, it might be indigenous and an opportunistic pathogen in fish.

\section{Acknowledgements}

This study was partly supported by Grants-in-Aid from JSPS and MEXT, Japan. We thank Dr. J. Bower, Hokkaido University, for his critical reading of this manuscript.

\section{References}

1) Aoki, T., T. Satoh and T. Kitao. 1987. New tetracycline resistance determinant of $\mathrm{R}$ plasmid from Vibrio anguillarum. Antimicriob. Agents Chemother. 31: 1446-1449.

2) Anderson, D.I. and B.R. Levin. 1999. The biological cost of antibiotic resistance. Curr. Opin. Microbiol. 2: 489-493.

3) Baticados, M.C.L., C.R. Lavilla-Pitogo, E.R. Cruz-Lacierda, L.D. de la Pena and N.A. Sunaz. 1990. Studies on the chemical control of luminous bacteria Vibrio harveyi and $V$. splendidus isolated from diseased Penaeus monodon larvae and rearing water. Dis. Aquat. Org. 9: 133-139.

4) Berthon, G., M. Brion and L. Lambs. 1983. Metal iontetracycline interactions in biological fluids; Part 2. potentiometric study of magnesium complexes with tetracycline, oxytetracycline, doxycycline, and minocycline, and discussion of their possible influence on the bioavailability of these antibiotics in blood plasma. J. Inorg. Biochem. 19: 1-18.

5) Chandrasekaran, S., B. Venkatesh and D. Lalithakumari. 1998. 
Transfer and expression of a multiple antibiotic resistance plasmid in marine bacteria. Curr. Microbiol. 37: 347-351.

6) Choi, M.S. 2000. Drug resistance of bacterial flora isolated from Kunsan Bay (In Korean). J. Fish. Pathol. 13: 111-119.

7) Choi, M.S., H.B. Shim and K.M. Joung. 2000. The distribution of bacterial flora in Kunsan bay (In Korean). J. Fish. Pathol. 13: 6773.

8) Chopra, I. and M. Roberts. 2001. Tetracycline antibiotics: mode of action, applications, molecular biology, and epidemiology of bacterial resistance. Microbiol. Mol. Biol. Rev. 65: 232-260.

9) Lambs, L., M. Venturim, B. Decock-Le Révérend, B. Kozlowski and G. Berthon. 1988. Metal ion-tetracycline interactions in biological fluids; Part 8. Potentiometric and spectroscopic studies on the formation of $\mathrm{Ca}(\mathrm{II})$ and $\mathrm{Mg}(\mathrm{II})$ complexes with 4dedimethylamino-tetracycline and 6-desoxy-6-demethy-tetracycline. J. Inorg. Biochem. 33: 193-210.

10) Levy, S.B. 1988. Tetracycline resistance determinants are widespread. ASM News 54: 418-421.

11) Levy, S.B. and M. Nelson. 1998. Reversing tetracycline resistance; a renaissance for the tetracycline family of antibiotics. p. 17-25. In Rosen and Mobashery (ed.), Resolving the antibiotic paradox. Kluwer Acadenic/Plenum Publishers, New York.

12) Lunestad, B.T. and J. Goksoyr. 1990. Reduction in the antibacterial effect of oxytetracycline in sea water by complex formation with magnesium and calcium. Dis. Aquat. Org. 9: 67-72.

13) Lunestad, B.T. and O.B. Samuelsen. 2001. Effects of sea water on the activity of antimicrobial agents used in aquaculture; implications for MIC testing. Aquaculture 196: 319-323.

14) McPhearson, R.M., A. Depaola, S.R. Zywno, Z.M. Motes Jr and A.M. Guarino. 1991. Antibiotic resistance in Gram-negative bacteria from cultured catfish and aquaculture ponds. Aquaculture 99: 203-211.

15) Nonaka, L. and S. Suzuki. 2002. New $\mathrm{Mg}^{2+}$-dependent oxytetracycline resistance determinant Tet 34 in Vibrio isolates from marine fish intestinal contents. Antimicrob. Agents Chemother. 46: $1550-1552$.

16) Nonaka, L., T. Isshiki and S. Suzuki. 2002. Distribution of the oxytetracycline resistance determinant Tet 34 among bacteria isolated from diseased fish. Microbes Environ. 17: 26-31.

17) Primavera, J.H., C.R. Lavilla-Pitogo, J.M. Ladja and M.R. de la Pena MR. 1993. A survey of chemical and biological products used in intensive prawn farms in the Philippines. Mar. Pollut. Bull. 26: 35-40.

18) Rhodes, G., G. Huys, J. Swings, P. McGann, M. Hiney, P. Smith and R.W. Pickup. 2000. Distribution of oxytetracycline resistance plasmids between aeromonads in hospital and aquaculture environments: implication of Tn1721 in dissemination of the tetracycline resistance determinant tet A. Appl. Environ. Micorbiol. 66: $3883-3890$.

19) Salyers, A.A., N.B. Shoemaker, A.M. Stevens and L-Y. Li. 1995. Conjugative transposons: an unusual and diverse set of integrated gene transfer elements. Microbiol. Rev. 59: 579-590.

20) Sambrook, J. and D.W. Russell. 2000. Molecular cloning: a laboratory manual, 3rd Ed. Cold Spring Harbor Laboratory Press, New York.

21) Schmidt, A.S., M.S. Bruun, I. Dalsgaare and J.L. Larsen. 2001. Incidence distribution and spread of tetracycline resistance determinants and integron-associated antibiotic resistance genes among motile aeromonads from a fish farming environment. Appl. Environ. Microbiol. 67: 5675-5682.

22) Schnappinger, D. and W. Hillen. 1996. Tetracyclines: antibiotic action, uptake, and resistance mechanisms. Arch. Microbiol. 165: 359-369.

23) Tendencia, E.T. and L.D. de la Peňa. 2001. Antibiotic resistance of bacteria from shrimp ponds. Aquaculture 195: 193-204.

24) Toranzo, A.E., P. Combarro, M.L. Lemos and J.L. Barja. 1984. Plasmid coding for transferable drug resistance in bacteria isolated from cultured rainbow trout. Appl. Environ. Microbiol. 48: 872-877.

25) Weisblum, B. 1995. Erythromycin resistance by ribosome modification. Antimicrob. Agents Chemother. 39: 577-585.

26) Weisburg, W.G., S.M. Barns, D.A. Pelletier and D.J. Lane. 1991. $16 \mathrm{~S}$ ribosomal DNA amplification for phylogenetic study. J. Bacteriol. 173: 697-703. 